\title{
Children born after intracytoplasmic sperm injection: population control study
}

\author{
Alastair G Sutcliffe, Brent Taylor, Jun Li, Simon Thornton, J Gedis Grudzinskas, Brian A Lieberman
} University College Medical School, Department of Child Health, Royal Free Campus, London NW3 2PF

Alastair G Sutcliffe, lecturer

Brent Taylor,

professor

Jun Li,

Centre for Assisted

Reproduction

(CARE), Park

Hospital,

Nottingham

NG5 8RX

Simon Thornton, medical director

Department of Obstetrics and Gynaecology, St Bartholomew's and Royal London School of Medicine and Dentistry, Royal London Hospital,

London E1 1BB

J Gedis

Grudzinskas,

professor

Department of

Reproductive

Medicine, St Mary's

Hospital,

Manchester

M13 0JH

Brian A Lieberman, medical director

Correspondence to: Dr Sutcliffe

icsi@rfhsm.ac.uk

BMJ 1999;318:704-5
Royal Free and

epidemiologist

Intracytoplasmic sperm injection is often successful for treatment of male infertility; over 20000 children have been born as a result. ${ }^{1}$ This bypassing of natural barriers to sperm selection has raised concerns about the children conceived. ${ }^{2}$ We report a population control study of children born in the United Kingdom as a result of this treatment.

\section{Subjects, methods, and results}

Children between 12 and 24 months old who had been singleton births were identified from a list of couples who had received the treatment and their parents were invited to participate; 123 of 137 families (90\%) agreed. Control children, conceived naturally, were recruited from associated nurseries $(105 / 123)$ or were social peers of cases (18/123). Altogether, 123 children born after intracytoplasmic sperm injection (study children) and 123 control children were seen. Children were matched for social class, maternal educational level, region, sex, and race but not maternal age. Multiple births were excluded to avoid confounding factors. Primary outcome measures were developmental scoring on the Griffiths scales of mental development ${ }^{3}$ and rates of congenital abnormalities. The Griffiths scales are an objective method of assessing development which uses five subscales. All subscales have a normal mean score of 100 (75-125, SD 1).

Clinical data obtained included date and type of delivery, birth weight, gestation, resuscitation required, duration and reason for admission to neonatal unit (if admitted), and details of ventilatory support. Congenital abnormalities were classed according to the ICD-10 (international classification of diseases, 10th revision). Sociodemographic data obtained included date of birth, sex, and age in months. Information obtained about parents included date of birth, social class, occupation, smoking status, alcohol intake, marital or support status (that is, the number of full time carers in the household), type of housing, education, race, and mother's gravidity and parity.

Chronological age and mean scores on Griffiths scales of mental development for infants conceived by intracytoplasmic sperm injection (study) and infants conceived naturally (control)

\begin{tabular}{|c|c|c|}
\hline & Study infants $(n=123)$ & Control infants $(n=123)$ \\
\hline & Mean (SD) score $(95 \% \mathrm{CI})$ & Mean (SD) score $(95 \% \mathrm{Cl})$ \\
\hline Chronological age (months) & $17.33(3.49)$ & $17.64(3.62)$ \\
\hline \multicolumn{3}{|c|}{ Griffiths scales of mental development } \\
\hline Griffiths quotient & 100.88 (13.52) (98.49 to 103.27) & 102.22 (11.53) (100.18 to 104.26) \\
\hline Mental age (months) & 17.26 (3.89) (16.57 to 17.95) & 17.99 (3.35) (17.46 to 18.59) \\
\hline \multicolumn{3}{|l|}{ Subquotients: } \\
\hline Locomotor & 96.47 (16.74) (93.51 to 99.43$)$ & 99.26 (12.92) (96.98 to 101.54) \\
\hline Personal and social & $99.20(14.70)(96.60$ to 101.80$)$ & 99.51 (14.06) (97.03 to 101.99) \\
\hline Hearing and speech & 108.98 (14.89) (106.35 to 111.61$)$ & 109.40 (13.42) (107.03 to 111.77) \\
\hline Eye-hand coordination* & 103.23 (15.53) (10.49 to 105.97$)$ & 106.77 (11.98) (104.65 to 108.89) \\
\hline Performance & 99.19 (12.12) (97.05 to 101.33) & 100.07 (13.31) (97.72 to 102.42) \\
\hline
\end{tabular}

${ }^{*}$ Analysis of variance with gestation taken into account, $\mathrm{P}<0.05$.
One observer (AGS) assessed all children. The mean age at assessment was 17.5 months. There was similarity across sociodemographic factors, although mothers of study children were more likely to be 35 years or older $(\mathrm{P}<0.001)$. Study children were more likely to have been born earlier (38.83 weeks $v 39.59$, $\mathrm{P}<0.01)$, to be of lower mean birth weight (3167 $\mathrm{g} v$ $3365 \mathrm{~g}, \mathrm{P}<0.01)$, and to have been born by caesarean section (44 v26, $\mathrm{P}<0.05)$, but neonatal admission rates were similar $(\mathrm{P}>0.7)$.

The mean mental age (17.3 months for study infants $v 17.6$ for controls) and the mean Griffiths quotient (101 for study infants $v 102$ for controls) were comparable (table). Difference in the eye-hand coordination subquotient persisted despite adjustment for gestation $(\mathrm{P}<0.05)$. However, scores on all subscales were normal for control and study children.

The number of study infants with minor congenital anomalies (14) was higher in comparison with controls (9), but not significantly. The number of children with a major congenital malformation was comparable 6 study $v 5$ controls). Malformations found in the study infants were scrotal fusion, undescended testis, exomphalos, congenital cataract, and congenital hip dislocation. In the control group malformations were buphthalmos, horseshoe kidney, cleft lip, cleft palate, and ventricular septal defect.

\section{Comment}

In our study a narrow age range was used to match cases and controls for assessment with the revised Griffiths scales (standardised on the UK population), which have a $91.3 \%$ power to detect a five point difference between groups. Children conceived naturally were chosen as controls in preference to another in vitro fertilisation group as being a more appropriate standard by which to test normal development. Control children were not matched by parity, history of infertility, or mode of delivery, which may be predictive of adverse outcome in later childhood. Our $90 \%$ follow up compares favourably with $25 \%$ in a Belgian study. ${ }^{4}$ Spontaneous abortions during the study period were not documented, possibly distorting the rates of congenital abnormality.

The difference in eye-hand coordination subscales is unlikely to be of functional significance; scores in both groups were normal. Congenital abnormality rates were consistent with national data in the United Kingdom (overall rate $5 \%)^{5}$ but a larger study might identify a true increase in minor anomalies.

We are recruiting further children, and follow up at 5 years of age is planned.

We would like to thank the following participating centres: Assisted Conception Unit, Ninewells Hospital, Dundee; BUPA Roding Hospital, Essex; Holly House Hospital, Essex; Churchill Clinic London; Lister Hospital, London; Midland Fertility Services, Birmingham; Manchester Fertility Services, St Mary's Hospital, Manchester; NURTURE, Department of Obstetrics and 
Gynaecology, Nottingham University; CARE, Park Hospital, Nottingham; General Infirmary at Leeds; Assisted Conception Unit, St James's Hospital, Leeds; and London Women's Clinic, Assisted Conception Unit, University College Hospital. Children from the following nurseries acted as controls: Tiger Tots (Aberdeen); University of Nottingham Crèche; Queen's Medical Centre Nottingham Crèche; the Surgery, 2 Ritchie Street, London; and the Mouse Hole Nursery (Middlesex Hospital Nursery). We are grateful for the advice of Dr Kerryn Saunders, Monash University, Melbourne, Australia.

Contributors: All authors participated in the design of this study, recruitment of patients, analysis of data, and writing of the paper. AGS assessed each child and is guarantor for the study.JL undertook the statistical analysis. BT advised on epidemiological aspects of the design of the study. ST, JGG, and BAL arranged the recruitment of patients and assisted with ethical approval and raising funding.

Funding: The study was supported by a grant from the Sir Halley Stewart Trust Fund. Additional grants were received from the Manchester Fertility Services Trust, the Burgess Bequest, Cook UK, Serono UK, Organon Laboratories, Ferring UK, IBSA Biochemique, and Smith Industries.

Competing interests: None declared.

1 De Mouzon J, Lancaster P. World collaborative report on in vitro fertilisation: preliminary data for 1995. J Assisted Reprod Genet 1997;14(suppl): 251-265S

2 Bowen JR, Gibson FL, Garth LI, Saunders DM. Medical and developmental outcome at one year for children conceived by intracytoplasmic sperm injection. Lancet 1998;351:1529-34.

3 Griffiths R. The Griffiths mental development scales 1996 revision. Henley: Association for Research in Infant and Child Development, Test Agency, 1996. (Revised by M Huntley.)

4 Bonduelle M, Joris H, Hofmans K, Liebaers I, Van Steirteghem AC. Mental development of 201 ICSI children at 2 years of age. Lance 1998;351:1553.

5 Botting B. Congenital anomalies. In: The health of our children. London: HMSO, 1997:148-58.

(Accepted 9 November 1998)

\section{Science commentary: what is involved in intracytoplasmic sperm injection?}

Intracytoplasmic sperm injection is a technique that increases the chance of conception in cases of severe male infertility or in cases in which the reasons for infertility remain unexplained. There are 59 centres in the United Kingdom licensed to conduct intracytoplasmic sperm injection.

Intracytoplasmic sperm injection involves injecting a single sperm into the centre of an ovum using a fine glass needle (one fourteenth the size of a human hair). The sperm can be collected from ejaculate or directly from the testis or epididymis; collection from the testis or epididymis is usually performed under general anaesthesia. Just before injection, the sperm is carefully prepared by washing off the seminal plasma; the best quality sperm are identified by washing through a special filter. Abnormally shaped sperm and those with poor motility are rejected. The chosen sperm is then concentrated in a tiny volume of culture fluid.

The woman undergoes ovarian stimulation, and then has ova collected as with in vitro fertilisation. The cumulus cells that surround the ovum are removed. The selected sperm is immobilised just prior to injection. Without this step, the ovum could be damaged by the beating of the tail of the sperm. It takes just a minute to inject the sperm directly into the ovum. Although the steps taken to obtain ova are the same as in routine in vitro fertilisation, intracytoplasmic sperm injection replaces the insemination stage. About 65\% of the ova injected are likely to be fertilised normally but, as with in vitro fertilisation, some will fail to develop and some embryos may not be of good quality. Placement of the embryo in the woman occurs in the same manner as for in vitro fertilisation.

Intracytoplasmic sperm injection can be used to overcome most cases of male infertility, even those in which the cause of infertility is unknown. Sperm injection can also be used to treat cases of infertility that have a genetic cause such as a chromo- somal abnormality-chiefly translocations or severe Klinefelter's syndrome; genetic causes account for up to $10 \%$ of problems with sperm. This method can also be used to treat carriers of cystic fibrosis, who may not have a vas deferens (Tuerlings JH et al, Fertility and Sterility 1998;69:899-903).

Sperm injection has also proved useful for men with testicular failure. Many men who have been diagnosed with testicular failure actually have tiny islands of normal spermatogenesis in their testes. By taking multiple fine needle aspirate biopsies of a testis to identify these islands, and by using the micromanipulation techniques of intracytoplasmic sperm injection to obtain their sperm, many men who have previously been told that they were infertile have managed to father children.

Although sperm injection offers hope for these men, there is concern that any genetic abnormality which may be as yet unidentified could unwittingly be passed on to their offspring. For this reason, centres that offer sperm injection recommend careful genetic counselling before a couple goes ahead with the procedure. The procedure itself could also carry a small risk. Since it involves piercing the outer and inner membranes of the ovum, and introducing a needle into the ovum, it is possible that the development of the embryo may be disturbed (Storeng RT et al, Acta Obstetricia et Gynecologica Scandinavica 1998;77: 191-7).There is also an increased risk of chromosomal abnormalities in the sperm of men who have problems with their sperm. Both of these factors may have contributed to early findings that the risk of abnormalities in the sex chromosomes (which can cause infertility) is increased by about $0.5 \%$ in offspring born as a result of intracytoplasmic sperm injection (Bonduelle $\mathrm{M}$ et al, Human Reproduction 1996;11:131-55).

Abi Berger Science editor, BMJ 